\title{
Macrophages and Angiogenesis in Human Gastrointestinal Tumors
}

\author{
P. Tonino*, E. Montero*, H. J. Finol*, F. Sánchez**, C. Hidalgo***, L. Sosa**** \\ *Center for Electron Microscopy, Faculty of Sciences, Central University of Venezuela, Apdo. \\ 76963, El Marquez 1070, Caracas, Venezuela \\ **Electron Microscopy Laboratory, Microbiology and Cell Biology Center, Venezuelan Institute for \\ Scientific Research, Venezuela \\ ***Structural Biology Department,Venezuelan Institute for Scientific Research, Venezuela \\ $* * * *$ Histology Department, Faculty of Medicine, Central University of Venezuela
}

Macrophages are members of the mononuclear phagocyte system and synthesize and release many angiogenesis stimulators (VEGF, bFGF, TGF- $\alpha$, PDGF) and inhibitors (TSP-1, interferon- $\alpha$, TGF- $\beta$, IL-1) [1,2]. Previous studies have demonstrated the importance of macrophages in tumor angiogenesis [2]. Whereas the presence of the extensive tumor-associated macrophages (TAM) infiltration has been shown to correlate with poor prognosis in a variety of human tumors, little is known about their role in gastrointestinal carcinomas. Te aim of this study was to analyze the macrophage number in relation to MVD and compare macrophage number in different regions of human gastrointestinal adenocarcinomas.

Specimens from the center of the tumor, periphery and lymph node metastasis were obtained immediately after surgical removal, from thirteen patients 44 to 76 years (mean 61.92 years) with a histopathological diagnosis of gastrointestinal adenocarcinoma. Measurements of MVD were performed and assessed previously in "hot spots" areas selected from Factor VIII/vWF immunostained sections [3]. Paraffin sections of the same regions were immnunostained using antihuman CD68 antibody (monoclonal, 1/50 dilution) by indirect immunoperoxidase technique. All positively stained macrophages $/ \mathrm{mm}^{2}$ were counted in 8 fields at $\mathrm{x} 250$ magnification using a graticule (defined area of $0.15 \mathrm{~mm}^{2}$ ), and values were expressed as mean \pm SEM. Tissue samples were also processed by routine transmission electron microscopy techniques and examined in Hitachi H-7100 and Philips CM10 electron microscopes. Statistical analysis were performed using STATISTICA package software. Statistical significance was defined as $P<0.05$.

A predominant diffuse pattern of CD68 immunostaining in the stroma of the tumor center and metastasis regions was observed, in contrast to the clusters present at the periphery of gastrointestinal adenocarcinomas (Fig. 1). Macrophages were present in areas surrounding tumor cells or blood vessels (Figs. 2), and also diffusely in some tumor areas, including the lumina of involved ducts. Macrophage number was $17.9 \pm 1.69$ macrophages $/ \mathrm{mm}^{2}$ at the tumor center region, $20.95 \pm 1.70$ at the periphery, and $16.75 \pm 2.33$ in the lymph node metastasis. Macrophages number did not differ between regions (ANOVA, $t$ test, $P>0.05$ ). No correlation between macrophages number and MVD was observed (Spearman rank test, $P>0.05$ ). At the ultrastructural level, morphology of macrophages was altered and they were found surrounding blood vessels (Fig. 3), as well as in direct contact with tumor cells.

Although macrophages are known to release a variety of angiogenics factors, this does not appear to be associated with MVD in human gastrointestinal adenocarcinomas, and may not mediate a 
proangiogenic role for macrophages in these tumors. The presence of perivascular macrophages could represent an acute response to blood vessel invasion by neoplastic cells.

References

[1] M. Orre and P.A.W. Rogers, Gynecol. Oncol.73 (1999) 47.

[2] N.J. Bingle et al., J. Pathol. 196 (2002) 254.

[3] P. Tonino, Acta Biol. Venez. 21 (2001) 1.

[4] This research was supported by a grant from CDCH of UCV (03.159.2001).
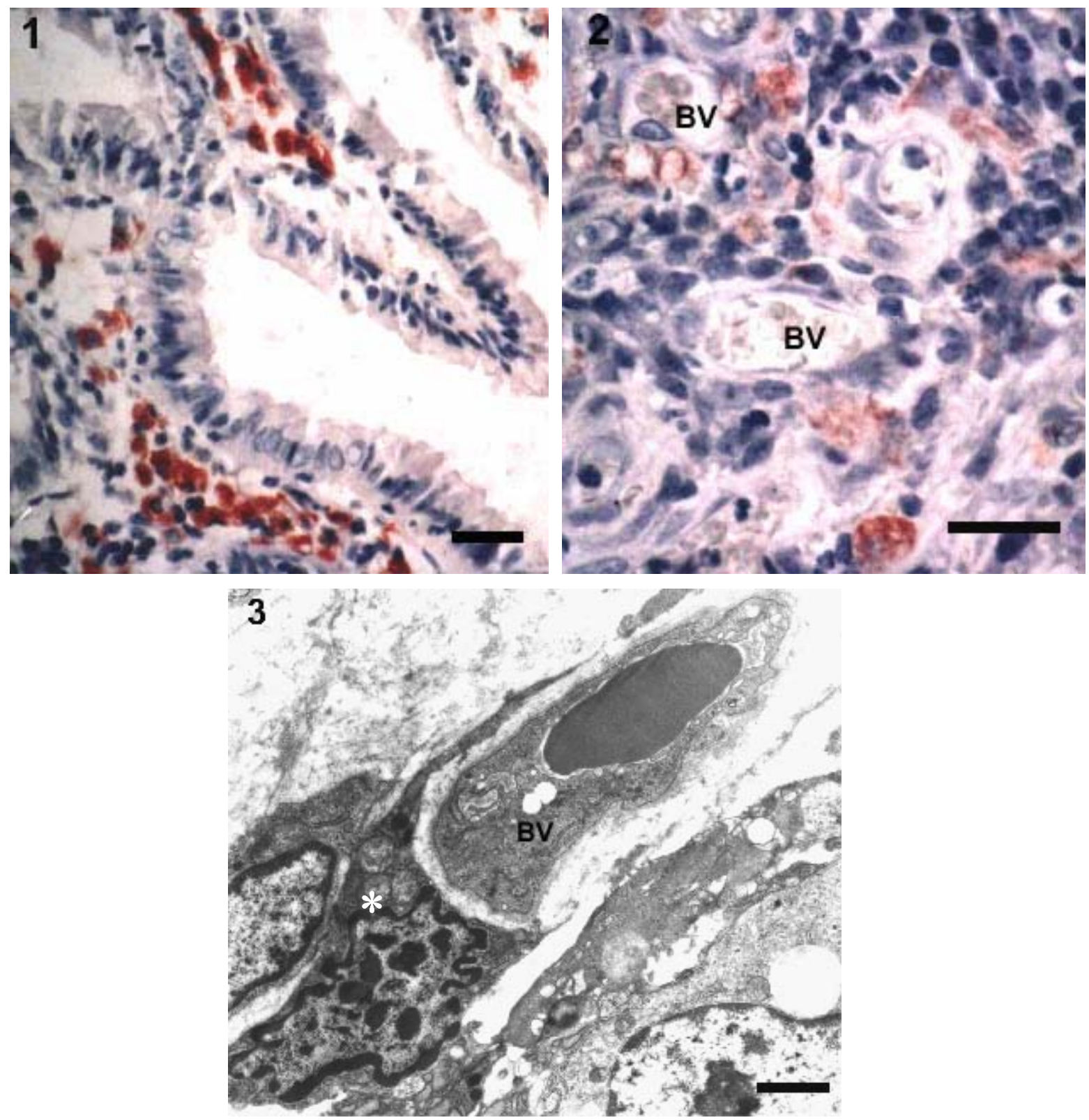

FIG. 1. Tumor-infiltrating macrophages stained with CD68 antibody. Scale Bar $=10 \mu \mathrm{m}$.

FIG. 2. Perivascular inflammation contains many macrophages (CD68). Scale Bar $=12.5 \mu \mathrm{m}$.

FIG. 3. Macrophage (asterisk) partially surrounding a blood vessel (BV). Scale Bar $=1 \mu \mathrm{m}$. 